\title{
Turbulence Induced Transport in Tokamaks
}

\author{
I. L. Caldas*, F. A. Marcus*, A. M. Batista , R. L. Viana**, S. R. Lopes**, M. V. A. \\ P. Heller*, Z. O. Guimarães-Filho*, P. J. Morrison* and W. Horton \\ ${ }^{*}$ Instituto de Física, Universidade de São Paulo, Caixa Postal 66318, 05315-970, São Paulo, SP, Brazil \\ ${ }^{\dagger}$ Departamento de Matemática e Estatística, Universidade Estadual de Ponta Grossa, Ponta Grossa, PR, Brazil \\ ${ }^{* *}$ Departamento de Física, Universidade Federal do Paraná, 81531-990, Curitiba, PR, Brazil. \\ Department of Physics and Institute for Fusion Studies, The University of Texas at Austin, Austin, Texas, 78712, \\ United States
}

\begin{abstract}
.
This report is concerned with plasma edge turbulence and its relation to anomalous particle transport in tokamaks. First, experimental evidence of turbulence driven particle transport and measurements of the gradients of the equilibrium profiles in the Brazilian tokamaks TBR and TCABR are presented. Next, diffusion in a two drift-wave system is discussed. In this nonintegrable system, particle transport is associated with the onset of chaotic orbits. Finally, numerical evidence suggesting that a nonlinear three-mode interaction could contribute to the intermittent plasma fluctuations observed in tokamaks is presented.
\end{abstract}

Keywords: plasma turbulence, anomalous transport, drift waves

PACS: $52.35 . \mathrm{Ra}, 52.35 . \mathrm{Kt}, 52.35 . \mathrm{Mw}$

\section{INTRODUCTION}

In tokamaks, the plasma is necessarily out of equilibrium due to the radial confinement. The observed particle flow limits the plasma confinement and remains as one of the key issues in tokamak research $[1,2]$.

The causes and the time rates of the cross-field transport have been one of the main themes in the study of plasma confinement in tokamaks [3]. Various theoretical models have been devised to identify the underlying plasma turbulence mechanisms thought to cause this anomalous transport $[2,4,5]$. Plasma turbulence often displays a broad fluctuation spectra with maxima at the longest measured scales (small wave vectors and high frequencies) [1].

In this work, we present experimental evidence of particle transport driven by the electrostatic turbulence observed in tokamaks. Moreover, we show that chaotic guiding center trajectories may significantly contribute to the observed particle transport. Furthermore, we present evidence that three-coupled unstable waves may generate intermittent fluctuations similar to those observed at the plasma edge in tokamaks.

\section{TRANSPORT INDUCED TURBULENCE IN TOKAMAKS}

In tokamaks, the plasma edge behavior depends on the anomalous particle transport caused by electrostatic turbulence. Langmuir probes measure fluctuations in the plasma edge parameters, such as density and plasma potential, and these measurements can be used to obtain the turbulence induced transport or to provide input for simulations like those presented in this work.

In this section we present some experimental results about the relationship between plasma transport and plasma turbulence, obtained in Ohmic discharges in the TBR and TCABR Brazilian tokamaks.

These measurements were performed with a multipin Langmuir probe system that provided simultaneous and local measurements of equilibrium and fluctuating values of density, potential, and temperature. For the TBR tokamak the major radius $R=0.30 \mathrm{~m}$, the plasma radius $a=0.08 \mathrm{~m}$, the toroidal field $B_{0}=0.40 \mathrm{~T}$, the plasma current $I_{p}=10 \mathrm{kA}$, and the plasma core density $n_{0} \approx 7 \times 10^{18} \mathrm{~m}^{-3}$. The radial profiles of the mean density, potential, and electron temperature have been measured (Fig. 1) [6, 7]. The relative density gradient, which has been identified as the turbulence energy reservoir $[1,2,8]$, can be estimated as

$$
\frac{1}{L_{N}}:=\left|\ln \left(\frac{n_{0}}{\omega_{c i}}\right)\right|=\left|\frac{\nabla n_{0}}{n_{0}}\right| \approx 167 m^{-1} .
$$

For an edge temperature $T_{e} \approx 10 \mathrm{eV}, \rho_{s}:=c_{s} / \omega_{c i}=$ $\sqrt{T_{e} / m_{i}} / \omega_{c i} \approx 10^{-3} \mathrm{~m}$, where $c_{s}$ is the sounds speed, $\omega_{c i}$ is the ion cyclotron frequency, and a working gas of Hydrogen is used. Below, frequencies are measured in units of $c_{S} / L_{N}$.

From two-point estimates we obtain poloidal wave number spectra, as well as mean wave number spectra, $\bar{k}$, and spectral widths, $\sigma_{k}$. A typical spectrum of potential 
fluctuations at $r / a=1.05$ (for a chosen time interval of $1.02 \mathrm{~ms}$ ) of TCABR [9, 10], which has $R=0.61 \mathrm{~m}$, $a=0.18 \mathrm{~m}, B_{0}=1.1 \mathrm{~T}, I_{P} \approx 100 \mathrm{kA}$ and $n_{0} \approx 1 \times 10^{19}$ $\mathrm{m}^{-3}$, is shown in Fig. 2. As can be seen in this figure, the plasma turbulence displays a broadband spectrum in both frequency and wave number. from

The fluctuation driven particle flux can be obtained

$$
\Gamma=\left\langle\tilde{n} \frac{\tilde{E}_{\theta}}{B_{0}}\right\rangle, \quad \tilde{E}_{\theta}=-\frac{1}{r} \frac{\delta \tilde{\phi}}{\delta \theta},
$$

where $\tilde{n}$ and $\tilde{E}_{\theta}$ are the fluctuating density and poloidal electric field, respectively. The particle flux at each frequency, $\Gamma(f)$, can be derived from the cross correlation between $\tilde{n}$ and $\tilde{\phi}$. Using this procedure, the following expression can be obtained [6]

$$
\Gamma(f)=\frac{2 \cdot k(f) \cdot\left|P_{\tilde{n} \tilde{\phi}}(f)\right| \cdot \sin \left[\theta_{\tilde{n} \tilde{\phi}}(f)\right]}{B_{0}}
$$

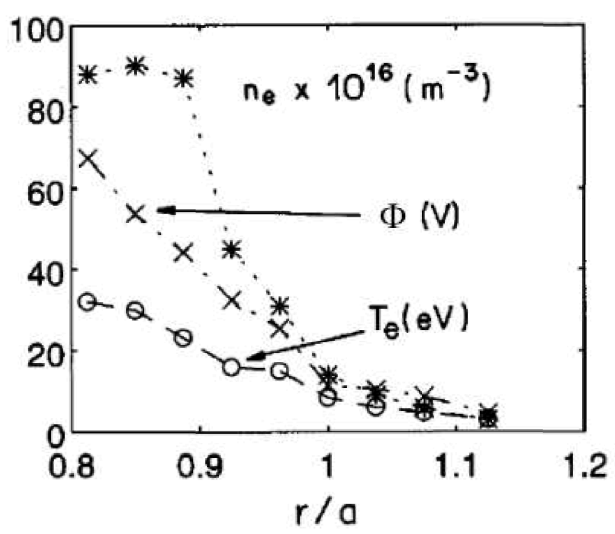

FIGURE 1. Edge plasma profiles of density, potential and temperature in the TBR tokamak (from [7]).

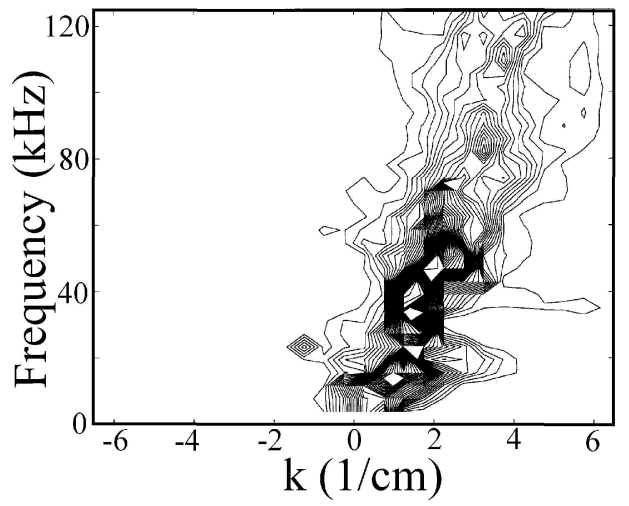

FIGURE 2. Contour plots of spectra, $S(k, f)$, for potential fluctuations at $r / a=1.05$. where $k(f)$ is the wave number of the fluctuating plasma potential, $P_{\tilde{n} \tilde{\phi}}(f)$ and $\theta_{\tilde{n} \tilde{\phi}}(f)$ are, respectively, the crosspower spectrum and the phase angle between the plasma potential and density fluctuations. Figure 3 shows the particle flux spectrum during a TCABR Ohmic discharge. As expected, this is also a broadband spectrum with the power concentrated on the observed electrostatic wave frequency and wave number range.

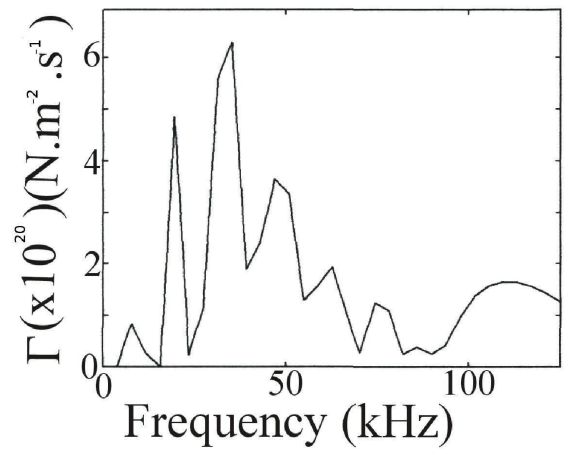

FIGURE 3. Induced particle transport spectra at $r / a=1.05$.

\section{TRANSPORT DUE TO LINEAR DRIFT WAVES}

Drift waves play a key role in our understanding of anomalous particle transport in tokamaks, where the presence of sufficiently steep density gradients in the plasma edge gives rise to drift waves and transport across the magnetic surfaces, i.e., a net flux of particles along the density gradient $[1,8]$.

The particle transport driven by these waves can be calculated considering the guiding center trajectories. We obtain these trajectories from the drift velocity of the guiding centers given by [11]:

$$
\vec{v}=\frac{\vec{E} \times \vec{B}}{B^{2}}
$$

where $\vec{E}=-\nabla \phi$. The guiding center equations are a Hamiltonian system with the Hamiltonian

$$
H(x, y, t)=\frac{\phi(x, y, t)}{B_{0}},
$$

where, as before, $\phi$ is the fluctuating plasma potential and $B_{0}$ is the toroidal field.

Here we consider the drift caused by two waves. Using dimensionless variables in a frame moving with the plasma phase velocity of the first wave, we obtain the hamiltonian

$$
\begin{aligned}
H(x, y, t)= & \left(u_{E}-u_{1}\right) x+A_{1} \sin \left(k_{x_{1}} x\right) \cos \left(k_{y_{1}} y\right)+ \\
& +A_{2} \sin \left(k_{x_{2}} x\right) \cos \left(k_{y_{2}}(y-u t)\right)
\end{aligned}
$$




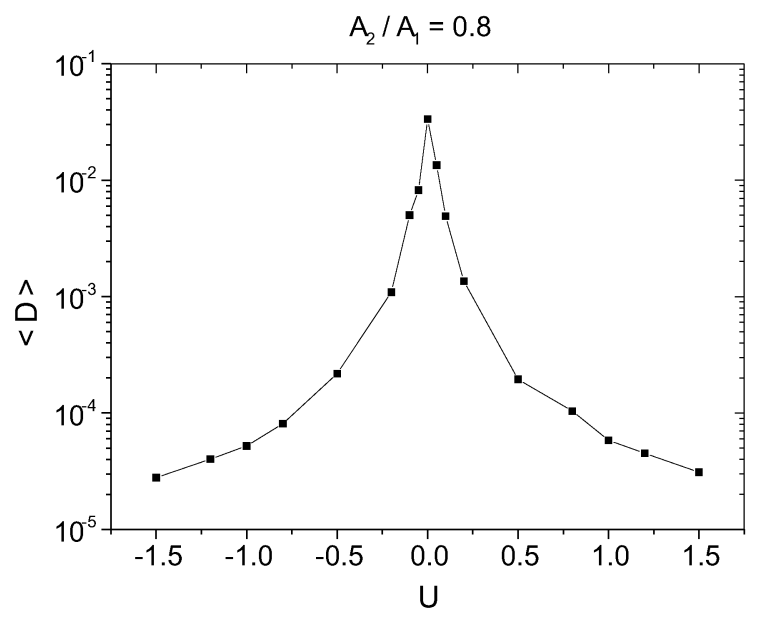

FIGURE 4. Diffusion coefficient as function of the trapping parameter for a fixed value $A_{2} / A_{1}=0.8$.

where $u_{E}:=E_{0} / B_{0}, u=\omega_{2} / k_{y_{2}}-\omega_{1} / k_{y_{1}}$ is the phase velocity difference between the second and the first waves, $u_{1}=\omega_{1} / k_{y_{1}}$, the term $\left(u_{E}-u_{1}\right) x$ is the equilibrium potential, and $x$ and $y$ correspond to the radial and poloidal coordinates, respectively.

When the system has only a single wave $\left(A_{2}=0\right)$, the Hamiltonian system is integrable and all guiding center trajectories are confined. In this case there is a single relevant dimensionless parameter called the trapping parameter $U$ :

$$
U=\frac{u_{E}-u_{1}}{A_{1} k_{x}} .
$$

However, for two waves, the system is not integrable and chaotic trajectories transport particles in the $x$ and $y$ directions. This effect can be confirmed by calculating the diffusion coefficient in the radial direction for a set of $N$ initial conditions $x_{i}(0)$ [12]:

$$
D(t)=\frac{1}{2 t N} \sum_{i=1}^{N}\left[x_{i}(t)-x_{i}(0)\right]^{2} .
$$

This diffusion coefficient depends strongly on the trapping parameter $U$. High values of $D$ are obtained for $U \approx 0$, when one of the phase velocities and the drift velocity are about the same (Fig. 4).

\section{TRANSPORT AND MODE COUPLING}

Because instabilities occur for a finite range of the spectrum, nonlinear mechanisms such as the nonlinear threemode interaction have been proposed to explain the broadband plasma turbulence observed in tokamak discharges $[14,15]$.

Hasegawa and Mima [16] derived a nonlinear partial differential equation for the drift-wave potential in order to explain the turbulence scenario produced in such a situation.

Horton and Hasegawa [1] considered a spectral decomposition for the Hasegawa-Mima equation, resulting in an infinite set of coupled ordinary differential equations for the complex Fourier mode amplitudes. They truncated this system by retaining three modes, subjected to a given resonance condition. The resulting system is intended to be a "building block" for obtaining the drift turbulence spectrum, in the sense that this system, although low-dimensional, is able to exhibit complex dynamics [17].

We introduced into the Horton-Hasegawa model phenomenological growth and decay rates of the three Fourier modes, so as to describe energy redistribution among different modes [13]. Such energy transfer processes are key for explaining the observed broadband spectrum of the drift-wave turbulence in the tokamak plasma edge [2]. In our numerical simulations we use growth and decay rates obtained from measurements of plasma edge turbulence [14].

Drift waves are low-frequency phenomena, and thus we assume the propagation of an electrostatic wave at a frequency $\omega$ much smaller than the ion cyclotron frequency $\omega_{c i}$. We assume the toroidal magnetic field $\mathbf{B}$ lies in the $z$-direction and assume that the phase velocity $\omega / k_{z}$ is less (greater) than the electron (ion) thermal velocity, with a scale length $\rho_{s}$ of the order of the ion cyclotron radius. In the drift-wave approximation it suffices to consider a cold ion plasma $\left(T_{i} \ll T_{e}\right)$ such that the ion dynamics are described by the fluid velocity $\mathbf{v}$. We also assume that within the length scale $\rho_{s}$, the quasineutrality hypothesis holds: $n \approx n_{e}=n_{0} \exp \left(e \phi / T_{e}\right)$.

In the presence of a density gradient, the electrostatic potential of the drift-wave, $\mathbf{E}=-\nabla \phi$, satisfies the Hasegawa-Mima equation

$$
\frac{\partial}{\partial t}\left(\nabla^{2} \phi-\phi\right)-[(\nabla \phi \times \hat{\mathbf{z}}) \cdot \nabla]\left[\nabla^{2} \phi-\ln \left(\frac{n_{0}}{\omega_{c i}}\right)\right]=0,
$$

where $\nabla$ is the gradient in the directions transversal to the magnetic field. The above equation involves only nondimensional quantities, since the coordinates $x$ and $y$ and time have been divided by the length scale $\rho_{s}$, time $t$ by the inverse of $\omega_{c i}$, and $\phi$ by $T_{e} / e$. The linearized Hasegawa-Mima equation allows plane wave solutions of the form $\phi=\phi_{0} \exp \left(i \mathbf{k} \cdot \mathbf{r}-i \omega_{k} t\right)$, where the drift-wave dispersion relation is

$$
\omega_{k}=-\frac{1}{1+k^{2}}\left[(\mathbf{k} \times \hat{\mathbf{z}}) \cdot \nabla \ln \left(\frac{n_{0}}{\omega_{c i}}\right)\right] .
$$

The partial differential equation (7) has an infinite number of degrees of freedom which, in Fourier space, are the electrostatic modes obtained by a Galerkin ex- 
pansion of the potential in the form

$$
\phi(\mathbf{x}, t)=\frac{1}{2} \sum_{k=1}^{\infty} \phi_{\mathbf{k}}(t) e^{i \mathbf{k} \cdot \mathbf{x}}+\text { c.c. },
$$

which when substituted into (7) results in an infinite number of coupled ordinary differential equations, from which three modes (we write $\phi_{j}=\phi_{\mathbf{k}_{j}}$ and $\omega_{j}=\omega_{\mathbf{k}_{j}}$ ) are retained:

$$
\begin{aligned}
& \dot{\phi}_{1}+i \omega_{1} \phi_{1}=\Lambda_{2,3}^{1} \phi_{2}^{*} \phi_{3}^{*}+\gamma_{1} \phi_{1} \\
& \dot{\phi}_{2}+i \omega_{2} \phi_{2}=\Lambda_{3,1}^{2} \phi_{3}^{*} \phi_{1}^{*}+\gamma_{2} \phi_{2} \\
& \dot{\phi}_{3}+i \omega_{3} \phi_{3}=\Lambda_{1,2}^{3} \phi_{1}^{*} \phi_{2}^{*}+\gamma_{3} \phi_{3},
\end{aligned}
$$

where the linear mode frequencies are given by ( 8 ) and, together with the wave numbers, satisfy resonance conditions

$$
\begin{aligned}
& \omega_{2}-\omega_{1}-\omega_{3} \approx 0 \\
& \mathbf{k}_{1}+\mathbf{k}_{2}+\mathbf{k}_{3}=\mathbf{0} .
\end{aligned}
$$

The coupling coefficients $\Lambda_{m, n}^{p}$ are determined by the wave numbers.

Because we are chiefly interested in describing the tokamak edge region, it is justifiable to use here an approximate rectangular geometry, where $x, y$, and $z$ stand for the radial position from wall, and the rectified poloidal and toroidal angles, respectively. Because $\mathbf{k}_{i z}$ is small, we set $\mathbf{k}_{i z}=0$, and so the resonance condition (14) defines a vector triangle in a plane perpendicular to the toroidal direction.

The numerical simulations we have performed used parameters values from the Brazilian tokamak TBR. The mean poloidal wave number of potential fluctuations was estimated at $k_{\theta} \sim 0.5 \mathrm{~cm}^{-1}$, and the spectrum of potential fluctuations is more pronounced at $\omega \sim 50 \mathrm{kHz}$. The other wave vector components were estimated assuming linear relations between them that obeying the resonance condition (14).

The phenomenological growth coefficients $\gamma_{i}$ were introduced by supposing that the "pump" mode, $\phi_{2}$, induces the process of energy redistribution among the "daughter" modes, $\phi_{1}$ and $\phi_{3}$. Hence we assigned a positive growth rate for mode 2 , whereas the modes 1 and 3 have negative growth rates. The numerical values for these growth rates were adjusted to fit potential fluctuations in the $-50 \mathrm{~V}$ to $+50 \mathrm{~V}$ range observed in experiments: $\gamma_{1}=\gamma_{3}<0$ and $\gamma_{2}=0.01$.

The three-mode truncation (10)-(12) of the Hasegawa-Mima equation is a flow in a six-dimensional phase space (since the Fourier modes are complex variables). The presence of growth rates makes this system a dissipative one, for which phase space volumes shrink in time with a constante rate $2\left(\gamma_{1}-\gamma_{2}-\gamma_{3}\right)<0$. Hence, we can expect that a large number of initial conditions would generate solutions of Eqs. (10)-(12), represented geometrically as trajectories in this phase-space, which asymptote to some attractor. The low-dimensional dynamics in this attractor can exhibit many interesting phenomena and some of them may be related to the generation of a fully-developed drift-wave turbulence that influences the anomalous transport at plasma edge.

In our numerical simulations, we have fixed all parameters but the decay rate of the daughter mode, $\gamma_{1}$, which we have taken as our control parameter. In the resonance condition (13) we allowed a small mismatch, and chose $\omega_{1}=\omega_{2}=\omega_{3}=1.31 \times 10^{-3}$. Representative examples of periodic and chaotic dynamics for the lowdimensional system can be obtained for different $\gamma_{1}$ values.

In Fig. 5(a), for $\gamma_{1}=-0.211$, the basic mechanism of mode coupling is observed, with widely different periods between consecutive spiking events. This is confirmed by the phase space projection depicted in Fig. 5(b), which apparently shows a chaotic attractor (in fact, the maximum Lyapunov exponent is positive for the trajectories on this attractor, signaling exponential separations for initial conditions very close to each other). The dynamics is strongly affected by the decay rate of the daughter modes. A further dimensional reduction is possibly by making a stroboscopic map with the discrete-time variable $z_{n}:=\max \left|\phi_{2}\right|$, which is obtained by sampling the local maxima in the time series of $\left|\phi_{2}\right|$. This procedure makes it possible to analyze the changes in the asymptotic dynamics of the system as the control parameter $\gamma_{1}$ is varied.

Figure 6 illustrates, for $\gamma_{1}=-0.192665$, another chaotic fluctuation, but the time series reveal that the chaotic behavior switches in an intermittent way to a period- 6 motion which, after $\gamma_{1}$ reaches the critical value $\gamma_{c}$, becomes stationary with no further chaotic bursts.

The average time between two consecutive bursts scales with the distance to the bifurcation point $\left|\gamma_{1}-\gamma_{c}\right|$ in the same way as predicted by the theory of type-I intermittency [19].

\section{CONCLUSIONS}

Experimental evidence in the TBR and TCABR tokamaks was reviewed and shown to provide evidence that particle transport is driven by electrostatic turbulence. Particle transport in a two drift-wave system was calculated from the guiding center chaotic trajectories and the radial diffusion coefficient was seen to depend strongly on the trapping parameter of (5).

Even though an infinite number of modes would be required to describe a fully-developed turbulent cascade, where energy is dissipated on ever smaller scales, results from low-dimensional dynamical systems such as 

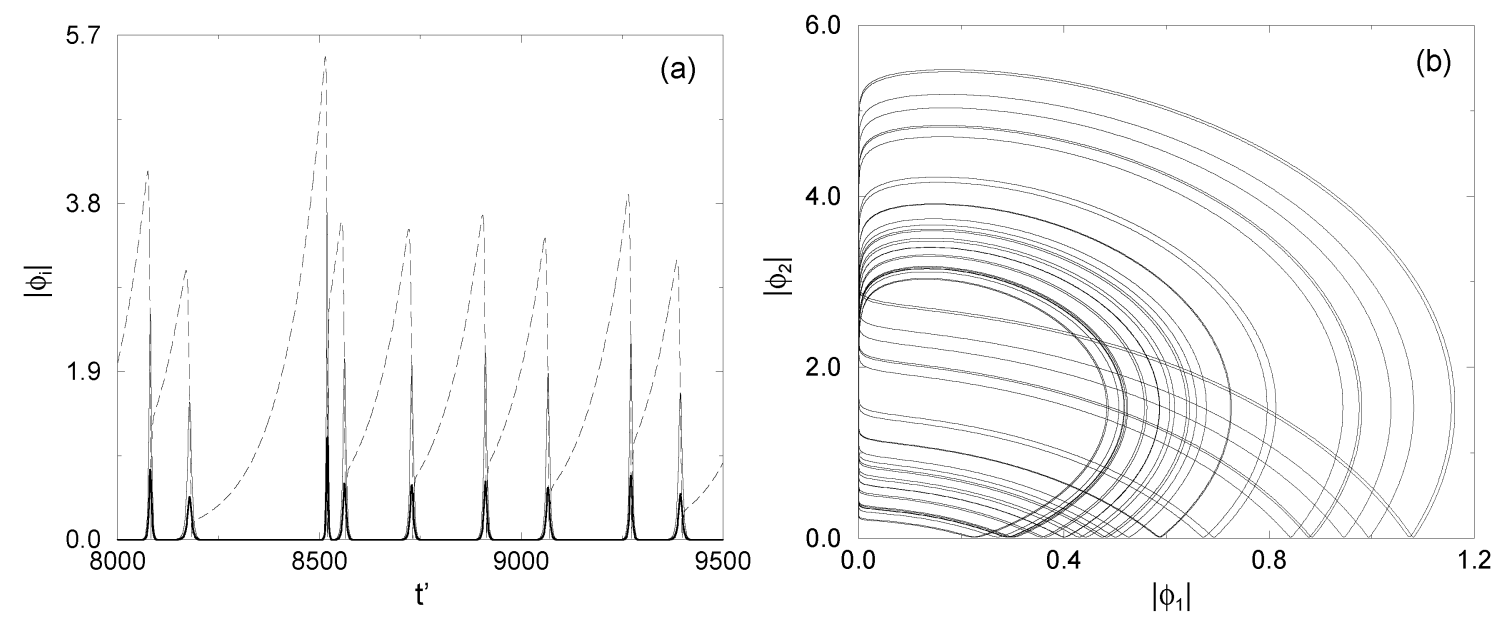

FIGURE 5. (a) Time series of the wave amplitudes $\left|\phi_{i}\right|[i=1$ (solid thin line), $i=2$ (dashed line), and $i=3$ (solid thick line) $]$ for $\gamma_{1}=-0.211$. (b) Phase space projection $\left|\phi_{2}\right|$ versus $\left|\phi_{1}\right|$.

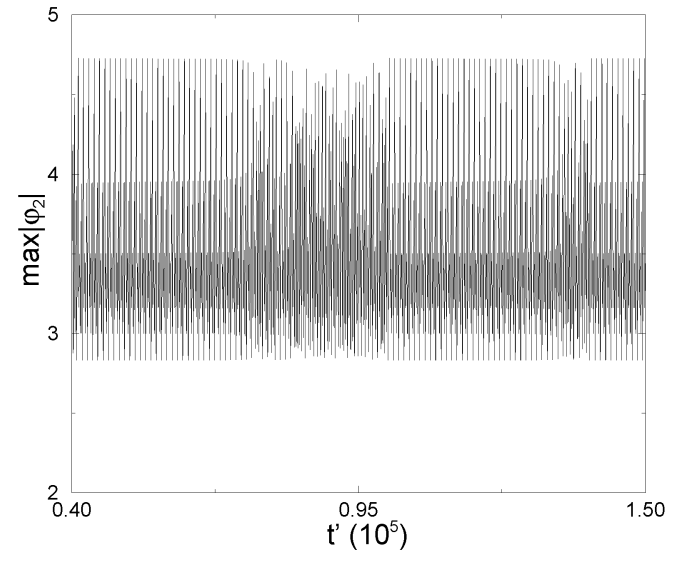

FIGURE 6. Time series of the variable $z_{n}:=\max \left|\phi_{2}(t)\right|$ for $\gamma_{1}=-0.192665<\gamma_{c}$, illustrating an intermittent alternation between laminar periodic oscillations and chaotic bursts.

those treated here may shed some light on the mechanisms leading to plasma turbulence. For example, in our three-wave model the energy goes from a strongly linearly unstable mode, the pump mode, to two daughter modes, with frequencies less and greater than the pump mode. Other modes can be introduced but, in view of the resonance condition (13), they would enter the model in triples, so our three-mode system might be regarded as a building block of a more complete theory.

The intermittency scenario we have described, in a special situation of temporal dynamics, is closely related to the so-called spatio-temporal intermittency, for which the switching between laminar and bursting behavior occurs for both temporal and spatial scales. Moreover, spatio-temporal intermittency is widely recognized as a possible scenario for the transition to fully-developed turbulence [20]. This opens an exciting road for future theoretical investigations.

\section{ACKNOWLEDGMENTS}

This work was partially supported by CNPq and CAPES (Brazilian Government Agencies), FAPESP (São Paulo State Agency), and two of us (PJM and WH) were supported by the U.S. Department of Energy under contract no. DE-FG03-96ER-54346.

\section{REFERENCES}

1. W. Horton, Rev. Mod. Phys. 71, 735 (1999).

2. A. J. Wootton, S. C. McCool, and S. Zheng, Fus. Technol. 19, 973 (1991). F. Wagner, and U. Stroh, Plasma Phys. Contr. Fusion 35, 1321 (1993).

3. R. D. Hazeltine and J. D. Meiss, Plasma Confinement (Addison Wesley, 1992).

4. R. Balescu, Transport Processes in Plasmas: Classical Transport Theory (Elsevier, Amsterdam, 1988)

5. S. J. Camargo, B. D. Scott, and D. Biskamp, Phys. Plasmas 3, 3912 (1996). S. J. Camargo, M. K. Tippett, and I. L. Caldas, Phys. Plasmas 7, 2849 (2000).

6. M. V. A. P. Heller, R. M. Castro, I. L. Caldas, Z. A. Brasilio, R. P. Silva, and I. C. Nascimento, J. Phys. Soc. Japan 66, 3453 (1997). M. V. A. P. Heller, Z. A. Brasilio, I. L. Caldas, J. Stöckel, and J. Petrzilka, Phys. Plasmas 6, 846 (1999).

7. R. M. Castro, M. A. V. Heller, I. L. Caldas, R. P. da Silva Z. A. Brasilio, and I. C. Nascimento, Phys. Plasmas 3, 971 (1996).

8. I. L. Caldas and H. Tasso, Plasma Phys. Contr. Fusion 20, 1299 (1978).

9. I. C. Nascimento, Y. K. Kuznetsov, A. M. M. Fonseca, A. Elfimov, V. Bellintani, M. Machida, M. V. A. P. Heller, 
R. M. O. GalvŃo, E. K. Sanada and J. I. Elizondo, Nucl. Fusion 45, 796 (2005).

10. M. A. V. Heller, I. L. Caldas, A. A. Ferreira, E. A. O. Saettone, A. Vannucci, I. C. Nascimento, and J. H. F. Severo, Czech. J. Phys. 55, 265 (2005).

11. W. Horton, Plasma Phys. Contr. Fusion 27, 937 (1985).

12. I. Osipenkov, W. Horton, and P. J. Morrison, Institute for Fusion Studies Report IFSR\#885 (2000), The University of Texas at Austin.

13. P. Terry and W. Horton, Phys. Fluids 26106 (1983); 25, 491 (1982); A. M. Batista, I. L. Caldas, R. L. Viana, S. R. Lopes, W. Horton, and P. J. Morrison, 'Nonlinear three-mode interaction and drift-wave turbulence in a tokamak edge plasma", to be published.

14. Ch. P. Ritz, E. J. Powers, T. L. Rhodes, R. D. Bengtson, K. W. Gentle, H. Lin, P. E. Phillips, A. J. Wootton, D. L. Brower, N. C. Luhman Jr., W. A. Peebles, P. M. Schoch, and K. L. Hickok, Rev. Sci. Instr. 59, 1739 (1988).

15. J. S. Kim, R. J. Fouck, R. D. Durst, E. Fernandez, P. W. Terry, S. F. Paul, and M. C. Zarnstortt, Phys. Rev. Lett. 79, 841 (1997).

16. A. Hasegawa and K. Mima, Phys. Rev. Lett. 39, 205 (1977); Phys. Fluids 2187 (1978).

17. J. M. Wersinger, J. M. Finn, and E. Ott, Phys. Rev. Lett. 44, 453 (1980); Phys. Fluids 23, 1142 (1980).

18. W. Horton and A. Hasegawa, Chaos 4, 227 (1994). P. Terry and W. Horton, Phys. Fluids 26106 (1983); 25, 491 (1982).

19. E. Ott, Chaos in Dynamical Systems (Cambridge University Press, Cambdrige, 1993).

20. H. Chaté and P. Manneville, Phys. Rev. Lett. 58 (1987) 112. H. Chaté, Europhys. Lett. 21 (1993) 419. 